Bull. Austral. Math. Soc.

$35 \mathrm{~K} 57,35 \mathrm{~B} 40,35 \mathrm{P} 10,35 \mathrm{Q} 40$

Vol. 48 (1993) [127-140]

\title{
PERIODIC SOLUTIONS AND GALERKIN APPROXIMATIONS TO THE AUTONOMOUS REACTION-DIFFUSION EQUATIONS
}

\author{
ARNold Dikansky
}

\begin{abstract}
The assumption that a Galerkin equation of the reaction-diffusion system of high order has an asymptotically orbitally stable time-periodic solution implies that the full reaction-diffusion system has a nearby asymptotically orbitally stable timeperiodic solution with asymptotic phase.
\end{abstract}

\section{INTRODUCTION}

In order to compute numerically solutions to the reaction-diffusion equation the first step is to replace the reaction-diffusion equation with a finite-dimensional Galerkin approximation. The Galerkin equations to the reaction-diffusion equation are finite dimensional systems which are obtained from the reaction-diffusion equation by projecting the equation on the linear space spanned by the first $m$ eigenfunctions of the Laplacian with corresponding boundary conditions and by truncating the remaining parts of the solutions in nonlinear terms. It is very desirable to relate the behaviour of solutions of the initial-boundary value problem for the reaction-diffusion equation to the behaviour of solutions of a finite-dimensional Galerkin approximation.

For the Navier-Stokes equations Constantin, Foias and Temmam [3] showed that if a Galerkin equation of high order, defined in terms of the Stokes operator, has an asymptotically stable stationary solution, then there exists a nearby asymptotically stable stationary solution to the Navier-Stokes equation. Titi [11] gave explicit applicable estimates for conditions established in [3]. Kloeden [8], using Lyapunov's second method, obtained a result similar to that of Constantin, Foias and Temam, considerably simplifing their proofs. Dikansky $[4,5]$ obtained a similar result for the reactiondiffusion equations using Lyapunov's second method as well as spectral properties of linear operators. Titi [12] obtained the existence of a stable time-periodic solution to the Navier-Stokes from the existence of a stable time-periodic solution to the Galerkin education of sufficiently high order. In [9] Kloeden considered relations between the existence of attractors and their stability properties for the Navier-Stokes equation and its Galerkin approximation.

Received 8 September 1992

Copyright Clearance Centre, Inc. Serial-fee code: 0004-9729/93 \$A2.00+0.00. 
Our aim here is to show that the existence of an orbital stable time-periodic solution with an asymptotic phase to a Galerkin equation of sufficiently high order for the autonomous reaction-diffusion system implies the existence of a nearby orbital stable time-periodic solution with an asymptotic phase to the boundary value problem for the reaction-diffusion system. To prove this it is shown (similarly to [4]) that the projection of a solution on the subspace $Q L_{2}$, complimentary to the finite-dimensional space $P L_{2}$ spanned by the first $m$ eigenfunctions of the operator $D \Delta$ with corresponding boundary conditions, is small if $m$ is large enough. Therefore the projection of the reactiondiffusion equation on $P L_{2}$ can be considered as a perturbed Galerkin equation. That allows the well-developed technique for the perturbed differential equation to be used to prove the existence of the periodic solution when the unperturbed ordinary differential equation has a noncritical periodic solution (see $[6,10,2])$. Finally the statement on orbital stability is proved.

\section{Preliminaries}

Throughout this article, the following notations will be used.

Let $\mathbb{R}^{n}$ denote Euclidean $n$-space. For $v \in \mathbb{R}^{n}$, let $\|v\|$ be any norm in $\mathbb{R}^{n}$. For an $n \times n$ matrix $A=\left(a_{i j}\right)$ define the norm of $A,\|A\|$ by $\|A\|=\sup _{\|v\|=1}\|A v\|$, where $v \in \mathbb{R}^{n}$.

Consider a system of ordinary differential equations

$$
\frac{d v}{d t}=F(v)
$$

Let $p(t)$ be a periodic solution of (1) with period $2 \pi$. Let $z=v-p$ and let the matrix with columns $\left(\partial F / \partial v_{i}\right)(p(t))$ be denoted by $F^{\prime}(p(t))$. Then

$$
\begin{aligned}
\frac{d z}{d t} & =F(z+p(t))-F(p(t)) \\
& =F^{\prime}(p(t))+g(t, z)
\end{aligned}
$$

where, by theorem of the mean, $g(t, z)=o(\|z\|)$ for small $\|z\|$. If $g$ is omitted from (2), we have the linear system

$$
\frac{d y}{d t}=F^{\prime}(p(t)) y
$$

which is called the variational equation with respect to the solution $p(t)$. The variational equation determines the nature of the stability of the solution $p(t)$ of (1). According to the Floquet theorem there exists a nonsingular transformation of variables $w=$ 
$P(t) y$ which transforms the linear periodic equation (3) into an equation with constant coefficients

$$
\frac{d w}{d t}=B w
$$

A monodromy matrix of (3) is a nonsingular matrix $C$ associated with a fundamental matrix solution $X(t)$ through the relation $X(t+2 \pi)=X(t) C\left(X(t)=P(t) e^{B t}\right)$. The eigenvalues $\rho$ of a monodromy matrix are called the characteristic multipliers of (3), and any $\lambda$ such that $\rho=e^{\lambda 2 \pi}$ are called characteristic exponents of (3).

If it assumed that $p(t)$ is a periodic solution of $(1), p^{\prime}(t)=F(p(t))$. On differentiating this equation, it follows that $p^{\prime}(t)$ is a solution of the variational equation (3). Thus the characteristic exponent associated with a solution of the linear system (3) may be taken as zero. The solution $v=p(t)$ may be regarded as a closed curve with $t$ as a parameter. If $n-1$ characteristic exponents of (3) have negative real parts, then the closed orbit is asymptotically stable in the sense that any solution of (1) which comes near a point of the orbit tends to the orbit as $t \rightarrow \infty$. This is called asymptotic orbital stability.

There is a generalisation of the Floquet theory on parabolic partial differential equations (see, for example [7]).

Let $\Omega$ denote an open bounded set of $\mathbb{R}^{n}$ with boundary $\Gamma$. For $(x, t) \in \Omega \times \mathbb{R}_{+}$ we consider the following reaction-diffusion system involving a vector function $u=$ $\left(u_{1}, u_{2}, \ldots, \ldots, n_{N}\right)$

$$
\frac{\partial u}{\partial t}=D \Delta u+F(u)
$$

Here $D=\operatorname{diag}\left(d_{1}, d_{2}, \ldots, d_{N}\right)$, where each $d_{j}>0$ is a constant and $F: \mathbb{R}^{N} \rightarrow \mathbb{R}^{N}$ is a $C^{2}$-function.

Equation (5) is supplemented with an initial condition

$$
u(x, 0)=u_{0}(x), \quad x \in \Omega
$$

and a boundary condition of either Dirichlet type or of Neumann type

$$
B u(x, t)=0, \quad x \in \Gamma, t \geqslant 0 .
$$

In the case of zero Dirichlet boundary conditions one imposes a compatibility condition $F(0)=0$.

A solution of the initial-boundary value problem (5), (6), (7) will be denoted by $u\left(x, t ; u_{0}\right)$. 
As usual $L^{\infty}(\Omega)$ will denote the space of functions defined on $\Omega$ which are bounded almost everywhere with norm $|\cdot|_{L^{\infty}}$. Let $H=L_{2}(\Omega)$ be the space of square integrable functions defined on $\Omega$ with the norm ||$_{H}$.

$\bar{P}$ will be used to denote the space of continuous, $2 \pi$-periodic functions from $\mathbb{R}$ into $\mathbb{R}^{n}$ with the norm: $\|g\|_{0}=\sup _{t}\|g(t)\|$, and $(u, v)$ is the usual inner product defined on vectors $u, v \in \mathbb{R}^{n}$.

Denote by $A$ the linear positive self-adjoint operator on $H$ given by $A u=-\Delta u$ supplemented by boundary conditions (7) with domain $D(A)=\left\{\phi \in W^{2,2}(\Omega),(7)\right.$ holds $\}$. Then $A$ is a sectorial operator and one can define the fractional powers $A^{\alpha}$ of $A, 0 \leqslant \alpha$ and the space $X^{\alpha}=D\left(A^{\alpha}\right)$ with the graph norm $|\cdot|_{X^{\alpha}}$. If $n \leqslant 3,3 / 4<\alpha<1$, then $X^{\alpha} \subset L^{\infty}(\Omega)$ with continuous inclusion:

$$
|v(\cdot)|_{L^{\infty}} \leqslant k|v(\cdot)|_{X^{\alpha}}, \quad v(x) \in X^{\alpha} .
$$

Denote by $P\left(0,2 \pi ; X^{\alpha}\right)$ the space of continuous, $2 \pi$-periodic in $t$ functions $g(x, t)$ for each $t$ belonging to $X^{\alpha}$ and equipped with the norm

$$
|g|_{X^{\alpha}, 0}=\sup _{t}|g(\cdot, t)|_{X^{\alpha}} .
$$

Since we are interested in the long-time behaviour of the solutions of (5) we modify the nonlinearity in equation (5) near $\infty$. Assume further that the nonlinear term $F(u)$ satisfies the following condition:

There is a $R_{1}>0$ such that

$$
F(u)=0 \quad \text { for } \quad|u|_{L^{\infty}} \geqslant R_{1} .
$$

Denote

$$
N_{1}=\sup \{|F(u)|\}, \quad N_{2}=\sup \left\{\left|F^{\prime}(u)\right|\right\}, \quad N_{3}=\sup \left\{\left|F^{\prime \prime}(u)\right|\right\} .
$$

One can then show that the initial-boundary problem for the reaction-diffusion equation (5), (6), (7) defines a local $C^{1,1}$ semigroup $S_{t}$ on $X^{\alpha}$ defined by $S_{t} u_{0}=$ $u\left(t, x ; u_{0}\right)$. For linear semigroups with generator $L$ we shall use the exponential notation $e^{L t}$. It is well known that the operator $A$ is self-adjoint as an operator in $L_{2}(\Omega)$ and the spectrum of $A$ consists of an infinite sequence of eigenvalues $0 \leqslant \lambda_{1} \leqslant \lambda_{2} \leqslant \ldots$. Because the matrix $D$ is diagonal, each eigenvalue of $A, \lambda_{i}$, corresponds to $N$ eigenvalues of the operator $D \Delta$ so there are $N$ eigenvalues $\lambda_{i}^{1}(D), \lambda_{i}^{2}(D), \ldots, \lambda_{i}^{N}(D)$ of the operator $D \Delta$ with corresponding eigenfunctions $\varphi_{i, D}^{1}(x), \varphi_{i, D}^{2}(x), \ldots, \varphi_{i, D}^{N}(x)$.

$$
\begin{aligned}
\Lambda_{i, D} & \stackrel{\text { def }}{=} \operatorname{diag}\left(\lambda_{i}^{1}(D), \lambda_{i}^{2}(D), \ldots, \lambda_{i}^{N}(D)\right), \\
\lambda_{i}(D) & \stackrel{\text { def }}{=} \min \left(\lambda_{i}^{1}(D), \lambda_{i}^{2}(D), \ldots, \lambda_{i}^{N}(D)\right),
\end{aligned}
$$


$(i=1,2, \ldots)$. Fix an integer $m$ and denote by $P_{m}$ the projection in $L_{2}(\Omega)$ onto the space spanned by the first $m$ eigenvectors of $A$, and we set $Q_{m}=I-P_{m}$. We recall that $P_{m}$ and $Q_{m}$ commute with $D \Delta$. If $u(x, t)$ is a solution of (5), (6), (7) we write $p=P_{m}, q=Q_{m}$, so that $u(x, t)=p(x, t)+q(x, t)$. Whenever it is possible we shall omit the index $m$. Let $X^{\alpha}=P X^{\alpha} \oplus Q X^{\alpha}$. Projecting equation (5) with boundary condition (7) on the invariant subspaces $P X^{\alpha}$ and $Q X^{\alpha}$, it is found that $p$ and $q$ are solutions for $t \geqslant 0$ of the coupled system of equations

$$
\begin{array}{ll}
\frac{d p}{d t}=\Lambda_{D} p+P F(p+q), & p(0)=p_{0}=P_{u_{0}} \\
\frac{\partial q}{\partial t}=D \Delta q+Q F\left(p_{q}\right), & q(0)=q_{0}=Q_{u_{0}} .
\end{array}
$$

Here $p(x, t)=\left(p^{1}(x, t), \ldots, p^{N}(x, t)\right)$, where $p^{k}(x, t)=\sum_{i=1}^{m} p_{i}^{k}(t) \varphi_{i, D}^{k}(x)$, $(k=1,2, \ldots, N)$, and $p_{i}^{k}(t)$ satisfies the following system of equations

$$
\frac{d p_{i}^{k}}{d t}=\Lambda_{D} p_{i}^{k}+\frac{1}{|\Omega|} \int_{\Omega} F^{k}\left(\sum_{i=1}^{m} p_{i}(t) \varphi_{i, D}(x)+q(x, t)\right) \varphi_{i, D}^{k}(x) d x .
$$

Using the variation of constant formula for (12) we rewrite equation (12) as

$$
q(x, t)=e^{L t} q_{0}(x)+\int_{0}^{t} e^{L(t-x)}[Q F(p+q)] d s .
$$

Here $e^{L t}$ is the linear semigroup corresponding to the problem

$$
\frac{\partial v}{\partial t}=D \Delta v
$$

in $Q X^{\alpha}$.

From [7, Theorem 1.5.4], the estimate

$$
\left|e^{L t} u(\cdot)\right|_{X^{\alpha}} \leqslant C_{1} e^{-\lambda_{m+1}(D) t}|u(\cdot)|_{X^{\alpha}}, \quad u(x, t) \in Q X^{\alpha}, \quad t>0,
$$

follows.

The Galerkin approximation to the reaction-diffusion equation (5) leads to the following system of ordinary differential equations

$$
\begin{aligned}
\frac{d u_{m}}{d t} & =\Lambda_{D} u_{m}+P_{m} F\left(u_{m}\right), \\
u_{m}(0) & =p_{0} \equiv u_{0} .
\end{aligned}
$$

It will be assumed that the Galerkin equation (16) possesses a periodic solution of period $\bar{T}$. To change the period $\bar{T}$ to $2 \pi$ we introduct the frequency $\bar{\omega}$ by setting $\bar{T}=2 \pi / \bar{\omega}$. After changing the independent variabe $t$ to $t / \bar{\omega}$, the Galerkin equation (16) becomes

$$
\bar{\omega} \frac{d u_{m}}{d t}=\Lambda_{D} u_{m}+P F\left(u_{m}\right) \text {. }
$$




\section{Main Result}

THEOREM . For every $\epsilon>0$ sufficiently small, there exists a natural number $M=$ $M(\epsilon)$ such that if the Galerkin equation (18) for some $m \geqslant M$ has an asymptotically orbitally stable time-periodic solution $\bar{u}_{m}(t)$ with frequency $\bar{\omega}, \bar{u}_{m}(t+2 \pi)=\bar{u}_{m}(t)$, then

(a) there exists a time-periodic solution $\bar{u}(x, t)$, with frequency $\omega$ of the boundary value problem for the reaction-diffusion equation (5), (7) such that

$$
\left|\bar{u}-\bar{u}_{m}\right|_{X^{\alpha}, 0} \leqslant C_{2} \epsilon, \quad|\omega-\bar{\omega}| \leqslant C_{3} \epsilon
$$

(b) the solution $\bar{u}(x, t)$ is asymptotically orbitally stable with asymptotic phase, that is if

$$
\min _{t}\left|u_{0}(x)-\bar{u}(x, t)\right|_{X^{\alpha}}<\rho, \quad \rho>0
$$

then the solution through $u_{0}(x)$ exists for all $t>0$ and, moreover, there exists $\theta^{*}>0$ such that

$$
\left|u\left(x, t ; u_{0}\right)-\bar{u}\left(x, t-\theta^{*}\right)\right|_{X^{\alpha}} \leqslant \rho e^{-\gamma t}, \quad \gamma>0, \quad t \geqslant 0 .
$$

ProOF: First consider the following linear periodic $q$-equation:

$$
\frac{\partial q_{1}}{\partial t}=\Delta q_{1}+f(x, t), \quad q_{1}(x, 0)=q_{1,0}(x)
$$

where $f(x, t) \in P\left(0,2 \pi ; X^{\alpha}\right),|f|_{X^{\alpha}, 0} \leqslant K$ and the constant $K$ does not depend on $m$.

Using the variation of constants formula a solution of the initial-boundary problem (21) is represented as

$$
q_{1}(x, t)=\epsilon^{L t} q_{1,0}(x)+\int_{0}^{t} e^{L(t-s)} f(x, s) d s .
$$

Since the solution $q_{1}(x, t)$ is periodic in $t$ with period $2 \pi$,

$$
q_{1,0}(x)=e^{L 2 \pi} q_{1,0}(x)+\int_{0}^{2 \pi} e^{L(2 \pi-s)} f(x, s) d s .
$$

Muliplying both sides of (23) by the inverse operator to the operator $\left[I-e^{L 2 \pi}\right]$ we find $q_{1,0}(x)$ :

$$
q_{1,0}(x)=\left[I-e^{L 2 \pi}\right]^{-1} \int_{0}^{2 \pi} e^{L(2 \pi-\mu)} f(x, s) d s
$$


Thus taking into consideration the estimate (15) one has from (24) the following estimate for $q_{1,0}(x)$ :

where

$$
\begin{gathered}
\left|q_{1,0}(\cdot)\right|_{X \alpha} \leqslant C_{4} K_{1} \zeta_{1}, \\
K_{1}=\int_{0}^{\infty} s^{-\alpha} e^{-\varepsilon} d s, \quad \zeta_{1}=L\left(\lambda_{m+1}(D)\right)^{-1},
\end{gathered}
$$

and the constant $C_{4}$ does not depend on $m$.

From (25) taking $M$ sufficiently large one obtains the following estimate for all $m \geqslant M$ :

$$
\left|q_{1,0}\right|_{X^{\alpha}} \leqslant \epsilon,
$$

where $\epsilon$ is a sufficiently small number.

The second term in (22) is estimated similarly. Therefore we have the following estimate for $\left|q_{1}\right|_{X^{a}, 0}$ :

$$
\left|q_{1}\right|_{X \propto, 0} \leqslant C_{5} \epsilon
$$

Because we want to prove the existence of a periodic solution of system (11), (12) of (unknown) period $T$ we introduce the (unknown) frequency $\omega$ by setting $T=2 \pi / \omega$. After changing the independent variable $t$ to $t / \omega$, system (11), (12) becomes

$$
\begin{aligned}
& \omega \frac{d p}{d t}=\Lambda_{D p}+P F(p+q), \\
& \omega \frac{\partial q}{\partial t}=D \Delta q+Q F(p+q),
\end{aligned}
$$

where now the existence of $\omega$ and a periodic solution of (28), (29), of fixed period $2 \pi$ is to be proved.

Let $p(t)=\bar{u}_{m}(t)+(\bar{\omega} / \omega) \bar{p}(t), \omega=\bar{\omega}+\beta$, in (28). Then if $\omega \neq 0, \bar{p}, \beta$ satisfy the following equation:

$$
\bar{\omega} \frac{d \bar{p}}{d t}=A\left(t, \bar{u}_{m}\right) \bar{p}+R(\bar{p}, q, t)-\beta \frac{d \bar{u}_{m}}{d t},
$$

where one used the assumption that $\bar{\omega}, \bar{u}_{m}(t)$ satisfy equation (18), and the following notations:

$$
\begin{aligned}
A\left(t, \bar{u}_{m}\right) w= & \Lambda_{D} w P\left[F_{\nu}\left(\bar{u}_{m}(x, t)\right) w\right], \\
R(\bar{p}, q, t)= & P F(p+q)-P F\left(\bar{u}_{m}\right)-P\left[F_{\nu}\left(\bar{u}_{m}\right) \bar{p}\right] \\
& +\frac{\bar{\omega}}{\omega} P\left[F_{\nu}\left(\bar{u}_{m}\right) p\right]-P\left[F_{\nu}\left(\bar{u}_{m}\right) q\right] .
\end{aligned}
$$


It will be proved that for some $\beta^{*}$, equation (28), (29) has a periodic solution $\bar{p}^{*}(t)$, $q^{*}(x, t)$ with $\bar{p}^{*}(t+2 \pi)=\bar{p}^{*}(t), q^{*}(x, t+2 \pi)=q^{*}(x, t)$.

Define $N \subset \mathbb{R} \times \bar{P} \times P\left(0,2 \pi ; X^{\alpha}\right)$, by

$$
N=\left\{(\beta, \bar{p}, q):|\beta| \leqslant \delta_{1},\|\bar{p}(t)\|_{0} \leqslant \delta_{2},|q|_{X^{\alpha}, 0} \leqslant \epsilon\right\},
$$

where it will be required that $\delta_{1}<\bar{\omega}$. Define a map $\mathcal{T}$ on the space $\mathbb{R} \times \overline{\boldsymbol{P}} \times$ $P\left(0,2 \pi ; X^{\alpha}\right), \mathcal{T}(\beta, \bar{p}, q)=\left(\beta_{1}, \bar{p}_{2}, q_{2}\right)$ as follows:

Given $(\beta, \bar{p}, q) \in N$, let $g_{1}(\beta, \bar{p}, q)=R(\bar{p}, q, t)$. Take

$$
\beta_{1}=\frac{1}{2 \pi} \int_{0}^{2 \pi}\left(g_{1}(\beta, \bar{p}, q)(s), v_{0}(s)\right) d s \cdot\left[\frac{1}{2 \pi} \int_{0}^{2 \pi}\left(\frac{d \bar{u}_{m}(s)}{d s}, v_{0}(s)\right) d s\right]^{-1},
$$

where $v_{0}(s),\left\|v_{0}\right\|_{2}=1$, denotes the unique periodic solution of the linear adjoint equation

$$
\omega \frac{d v_{0}}{d t}=-A^{*}\left(t, \bar{u}_{m}\right) v_{0} .
$$

Therefore

$$
g_{2}\left(\beta, \bar{p}, q, \beta_{1}\right)=g_{1}(\beta, \bar{p}, q)-\beta_{1} \frac{d \bar{p}}{d s}
$$

satisfies the orthogonality condition:

$$
\int_{0}^{2 \pi}\left(g_{2}\left(\beta, \bar{p}, q, \beta_{1}\right)(s), v_{0}(s)\right) d s=0,
$$

which guarantees the existence of a unique periodic solution of equation (36). Let $p_{2}, q_{2}$ denote a unique periodic solution of the following linear system:

$$
\begin{aligned}
& \bar{\omega} \frac{d p_{2}}{d t}=A p_{2}+g_{2}\left(\beta, \bar{p}, q, \beta_{1}\right), \\
& \bar{\omega} \frac{\partial q_{2}}{\partial t}=D \Delta q_{2}+g_{3}\left(\beta, \bar{p}, \beta_{1}\right)
\end{aligned}
$$

satisfying

$$
\begin{aligned}
\left\|p_{2}\right\|_{0} & \leqslant C_{6}\left\|g_{2}\right\|_{0}, \\
\left|q_{2}\right|_{X^{\alpha}, 0} & \leqslant \epsilon .
\end{aligned}
$$

Due to the assumption on the characteristic multipliers of the matrix $A\left(t, \bar{u}_{m}\right)$, the orthogonality condition (35) and estimate (27), such $p_{2}, q_{2}$ exist.

It is clear that $\mathcal{T}(\beta, \bar{p}, q)=\left(\beta_{1}, p_{2}, q_{2}\right) \in \mathbb{R} \times \bar{P} \times P\left(0,2 \pi ; X^{\alpha}\right)$. Now it will be shown that $(\beta, \bar{p}, q) \in N$ implies that $\left|\beta_{1}\right| \leqslant \delta_{1},\left\|p_{2}\right\|_{0} \leqslant \delta_{2},\left|q_{2}\right|_{X^{\alpha}, 0} \leqslant \epsilon$. From the definition of $g_{1}$ one obtains

$$
\left\|g_{1}(\beta, \bar{p}, q)\right\|_{0} \leqslant \frac{C_{7} \bar{\omega}^{2}}{\left(\bar{\omega}-\delta_{1}\right)^{2}}\left(\|\bar{p}\|_{0}\right)^{2}+C_{8} \epsilon+\frac{C_{9}}{\bar{\omega}-\delta_{1}}|\beta|\|\bar{p}\|_{0},
$$


where due to condition (9) the constants $C$ do not depend on $m$. From (33) and the Schwartz inequality it follows that

$$
\left|\beta_{1}\right| \leqslant C_{10}\left\|g_{1}(\beta, \bar{p}, q)\right\|_{0} .
$$

Therefore from (38), (40) one obtains the following estimates:

$$
\left|\beta_{1}\right| \leqslant \delta_{1}, \quad\left\|p_{2}\right\|_{0} \leqslant \delta_{2}, \quad\left|q_{2}\right|_{X^{\alpha}, 0} \leqslant \epsilon .
$$

Taking $M=M(\epsilon)$ large enough one has $\delta_{1}=\delta_{1}(\epsilon)$ small enough and therefore the mapping $\mathcal{T}$ maps $N$ into itself.

Now because the map $\mathcal{T}$ is a compact mapping of $\mathbb{R} \times \bar{P} \times P\left(0,2 \pi ; X^{\alpha}\right)$ into itself the existence of the desired fixed point $\beta^{*}, \bar{p}^{*}, q^{*}$ of equation (36), (37) follows from the Schauder theorem.

So the existence of a solution $\bar{u}=\bar{u}_{m}+\bar{p}^{*}+q^{*}, \omega=\bar{\omega}+\omega^{*}$ of equation (11), (12) with estimates (19) has been proved.

It is left to show that the solution $\bar{u}(x, t)$ is asymptotically orbitally stable with asymptotic phase. Changing the variables

$$
u(x, t)=\bar{u}(x, t)+z(x, t)
$$

applied to (5) yields

$$
\frac{\partial z}{\partial t}=D \Delta z+F^{\prime}(\bar{u}) z+g(t, z)
$$

where

$$
g(t, 0)=0, \quad\left|g\left(t, z_{1}\right)-g\left(t, z_{2}\right)\right| \leqslant k(\rho)\left|z_{1}-z_{2}\right|_{X^{\alpha}},
$$

if $\left|z_{1}\right|_{X^{\alpha}},\left|z_{2}\right|_{X^{\alpha}} \leqslant \rho$ as $\rho \rightarrow 0$. Consider the corresponding linearised equation

$$
\frac{\partial u_{1}}{\partial t}=D \Delta u_{1}+F^{\prime}(\bar{u}(x, t)) u_{1} .
$$

Let $U(t)$ denote the Poincare map, $U(t)=W(t+T, t), W(t, s)$ being the evolution operator of (45) and $T$ the period of the solution $\bar{u}(x, t)$. Note that because $\bar{u}(x, t)$ is the solution of the autonomous differential equation, $\{1\}$ is a spectral set of the spectrum $\sigma(U(t))$. By results of [7] we have the decomposition $X^{\alpha}=X_{1}(t) \oplus X_{2}(t)$, where $X_{1}(t)=\operatorname{span}\{\bar{u}(x, t)\}$ and

$$
\left.\sigma(U(t))\right|_{X_{1}(t)}=\sigma=\{1\},\left.\quad \sigma(U(t))\right|_{X_{2}(t)}=\sigma(U(t))-\sigma_{1},
$$

$X_{1}(t), X_{2}(t)$ are invariant and if $t \geqslant s$ we have $W(t, s): X_{1}(s)=X_{1}(t)$, is one-to-one and onto. 
Consider equation (45) on $X_{2}$ :

$$
\frac{\partial u_{2}}{\partial t}=D \Delta u_{2}+F^{\prime}(\bar{u}(x, t)) u_{2}
$$

Projecting equation (47) on the subspaces $P_{m} X_{2}, Q_{m} X_{2}$ one has the following system of equations

$$
\begin{array}{ll}
\frac{d p_{2}}{d t}=\Lambda_{D} p_{2}+P\left[F^{\prime}(\bar{u})\left(p_{2}(x, t)+q_{2}(x, t)\right)\right], & p_{2}(0)=p_{2,0}, \\
\frac{\partial q_{2}}{\partial t}=\Delta q_{2}+Q\left[F^{\prime}(\bar{u})\left(p_{2}(x, t)+q_{2}(x, t)\right)\right], & q_{2}(0)=q_{2,0}
\end{array}
$$

Using the variation formula for equation (49) and estimate (15) one deduces the following estimate

$$
\begin{aligned}
\left|q_{2}(\cdot, t)\right|_{X^{\alpha}} \leqslant & C_{1} e^{-\lambda_{m+1}(D) t}\left|q_{2,0}(\cdot)\right|_{X^{\alpha}} \\
& +C_{1} N_{2} \int_{0}^{t}(t-s)^{-\alpha} e^{-\lambda_{m+1}(D)(t-s)}\left|\left(p_{2}(\cdot, s)+q_{2}(\cdot, s)\right)\right|_{H} d s
\end{aligned}
$$

Defining for $0<\sigma<\lambda_{m+1}(D)$

$$
\bar{q}(t) \stackrel{\text { def }}{=} e^{\sigma t}\left|q_{2}(\cdot, t)\right|_{X^{\alpha}},
$$

from (21) for $\bar{q}(t)$ one deduces the estimate

$$
\begin{aligned}
\bar{q}(t) \leqslant & C_{1} e^{-\left(\lambda_{m+1}(D)-\sigma\right) t} \bar{q}(0) \\
& +C_{1} N_{2}|\Omega|^{1 / 2} \int_{0}^{t}(t-s)^{-\alpha} e^{-\left(\lambda_{m+1}(D)-\sigma\right)(t-s)} \bar{q}(s) d s \\
& +k C_{1} N_{2}|\Omega|^{1 / 2} e^{\sigma t} \int_{0}^{t}(t-s)^{-\alpha} e^{-\lambda_{m+1}(D)(t-s)} \widehat{p}_{2}(t) d s,
\end{aligned}
$$

where $\widehat{p}_{2}(t)=\sup _{0 \leqslant s \leqslant t}\left\|p_{2}(s)\right\|$. Therefore from $(51)$ for

$$
\breve{q}(t) \stackrel{\text { def }}{=} \sup _{0 \leqslant s \leqslant t} \breve{q}(s)
$$

one obtains for $0 \leqslant s \leqslant t$ that

$$
\begin{gathered}
\breve{q}(t) \leqslant C_{1} e^{\left(\lambda_{m+1}(d)-\sigma\right) t} \tilde{q}(0)+\zeta_{1} \breve{q}(t)+\zeta_{2} e^{\sigma t} \widehat{p}_{2}(t) \\
K_{2}=\int_{0}^{\infty} s^{-\alpha} e^{-\left(-\frac{\sigma}{\lambda_{m+1}(D)}\right)} d s \\
\zeta_{1}=k K_{2} C_{1} N_{2}|\Omega|^{1 / 2}\left(\lambda_{m+1}(D)\right)^{\alpha-1} \\
\zeta_{2}=k K_{1} C_{1} N_{2}|\Omega|^{1 / 2}\left(\lambda_{m+1}(D)\right)^{\alpha-1}
\end{gathered}
$$


Inequality (52) implies that

$$
\breve{q}(t)\left(1-\zeta_{1}\right) \leqslant C_{1} \widetilde{q}(0)+\zeta_{2} e^{\sigma t} \widehat{p}_{2}(t) .
$$

Therefore

$$
\left|q_{2}(\cdot, t)\right|_{X^{\alpha}} \leqslant \frac{C_{1} e^{-\sigma t}\left|q_{2,0}(\cdot)\right|_{X^{\alpha}}}{1-\zeta_{1}}+\frac{\zeta_{2}}{1-\zeta_{1}} \widehat{p}_{2}(t)
$$

if $\zeta_{1}<1$. So taking $M$ large enough $\zeta_{1}$ and $\zeta_{2}$ will be small enough and from (54) one deduces the estimate

$$
\left|q_{2}(\cdot, t)\right|_{X^{\alpha}} \leqslant C_{12} e^{-\sigma_{1} t}\left|q_{2,0}(\cdot)\right|_{X^{\alpha}}+C_{13} \eta \widehat{p}_{2}(t),
$$

where $\eta=O\left(\lambda_{m+1}^{-1+\alpha}(D)\right)$ is sufficiently small when $M$ is large enough.

We rewrite equation (48) as

$$
\begin{aligned}
\frac{d p_{2}}{d t} & =\Lambda_{D} p_{2}+P\left[F^{\prime}\left(\bar{u}_{m}\right) p_{2}\right]+P\left[\left(F^{\prime}(\bar{u})-F^{\prime}\left(\bar{u}_{m}\right)\right) p_{2}\right]+P\left[F^{\prime}(\widehat{u}) q_{2}\right] \\
p_{2}(0) & =p_{2,0} .
\end{aligned}
$$

Let $X(t)$ be the fundamental matrix solution of the linearised Galerkin equation:

$$
\frac{d u_{m, 2}}{d t}=\Lambda_{D} u_{m, 2}+P\left[F^{\prime}\left(\bar{u}_{m}\right) u_{m, 2}\right]
$$

The Floquet representation for the linearised Galerkin operator implies $X(t)=Y(t) e^{B t}$, where all eigenvalues of the constant matrix $B$ have negative real parts, and $Y(t)$ is a periodic nonsingular matrix with $\|Y(t)\|,\left\|Y^{-1}(t)\right\| \leqslant C_{14}$. Due to (19) the constant $C_{14}$ does not depend on $m$ for all $m \geqslant M$. Furthermore, the transformation $p_{2}=$ $Y(t) \bar{p}_{2}$ applied to $(56)$ yields

$$
\frac{d \bar{p}_{2}}{d t}=B \bar{p}_{2}+Y^{-1}(t)\left\{P\left[\left(F^{\prime}(\bar{u})-F^{\prime}\left(\bar{u}_{m}\right)\right) Y(t) \bar{p}_{2}\right]+P\left[F^{\prime}(\bar{u}) q_{2}\right]\right\}
$$

with $\mathcal{R} S_{p}(B) \geqslant \gamma_{1}>0$.

Because of (19) one has

$$
\left\|F^{\prime}(\bar{u})-F^{\prime}\left(\bar{u}_{m}\right)\right\| \leqslant N_{3}|\Omega|^{1 / 2} \epsilon .
$$

Applying the variation of constants formula for the equation

$$
\frac{d v}{d t}=B v
$$


to (58), and taking into consideration (55) one has

$$
\begin{aligned}
\left\|\bar{p}_{2}(t)\right\| \leqslant & C_{15} e^{-\gamma_{1} t}\left\|\bar{p}_{1,0}\right\|+\epsilon C_{16} \int_{0}^{t} e^{-\gamma_{1}(t-s)}\left\|\bar{p}_{2}(s)\right\| d s \\
& +C_{17} \int_{0}^{t} e^{-\gamma_{1}(t-s)}\left|q_{2}(\cdot, s)\right|_{X^{\alpha}} d s .
\end{aligned}
$$

From standard considerations on small perturbations of the resolvent (see for example, the proof of Theorem 1.3.2 in [7]) it follows that the constant $C_{15}$ (and therefore all other constants in (60)) does not depend on $m, m \geqslant M$. From (55) and (60) for

$$
\omega(t) \stackrel{\text { def }}{=} \sup _{0 \leqslant s \leqslant t} e^{\sigma_{2} t}\left\|\bar{p}_{2}(t)\right\|, \quad\left(0<\sigma_{2}<\sigma_{1}<\gamma_{1}\right) .
$$

one obtains the estimate

$$
\omega(t) \leqslant C_{15} e^{-\left(\gamma_{1}-\sigma_{2}\right) t} \omega(0)+\zeta_{3} K_{3} \omega(t)+C_{18} e^{-\left(\gamma_{1}-\sigma_{2}\right) t} K_{3}\left|q_{2,0}(\cdot)\right| x^{\alpha},
$$

with $\zeta_{3}$ small enough when $\epsilon$ is small enough and $M$ is large enough, and

$$
K_{3}=\int_{0}^{\infty} e^{-\left(\gamma_{1}-\sigma_{1}\right) \cdot} d s
$$

Because $\zeta_{3}$ is small enough inequality $(61)$ implies that

$$
\left\|\bar{p}_{2}(t)\right\| \leqslant C_{19} e^{-\sigma_{2} t}\left|u_{2}(\cdot, 0)\right|_{X^{\alpha}} .
$$

Substituting (62) in (55) we get

$$
\left|g_{2}(\cdot, t)\right|_{X^{\alpha}} \leqslant C_{20} e^{-\gamma_{2} t}\left|u_{2}(\cdot, 0)\right|_{X^{\alpha}}
$$

Finally from (62) and (63) we have

$$
\left|u_{2}(\cdot, t)\right|_{X^{\alpha}} \leqslant C_{21} e^{-\gamma_{3} t}\left|u_{2}(\cdot, 0)\right|_{X^{a}}, \quad \gamma_{3}>0 .
$$

Therefore we have the estimates for $v_{2} \in X_{2}$

$$
\left|W(t, s) v_{2}\right|_{X^{\alpha}} \leqslant C_{22} e^{-\gamma_{3}(t-s)}\left|v_{2}\right|_{X^{\alpha}}, C_{22}(t-s)^{-\alpha} e^{-\gamma_{3}(t-s)}|v|_{H},
$$

where $\gamma_{2}>0$. From (65) it also follows that the Poincare map $W$ has only one characteristic multiplier equal to 1 and all other characteristic multipliers have modulus less than 1 (characteristic exponents have negative real parts). 
Also, for $v_{1} \in X_{1}(s)$, there is $C_{23}$ such that we have the estimate

$$
\left|W(t, s) v_{1}\right|_{X^{\alpha}} \leqslant C_{23} e^{-\gamma_{4}(t-s)}\left\|v_{1}\right\|, \quad s \geqslant t
$$

with $\gamma_{4}>0$. This estimate follows from the finite dimensionality of $X_{1}(s)$.

We define the following map $z \rightarrow G(z)$ by

$$
\begin{gathered}
(G z)(t)=W(t, 0) \alpha+\int_{0}^{t} W(t, s) E_{2}(s) g(s, z(s)) d s \\
-\int_{t}^{\infty} W(t, s) E_{1}(s) g(s, z(s)) d s
\end{gathered}
$$

where $E_{1}, E_{2}$ are the projections associated with $X_{1}(s), X_{2}(s)$.

Choosing $\rho>0$ so small that

$$
C_{24} k(\rho) \bar{E}\left(\frac{1}{\gamma_{4}}+\int_{0}^{\infty} e^{-\left(\gamma_{3}-\gamma_{4}\right) s_{s}-\alpha} d s\right) \leqslant \epsilon_{1}
$$

with $\epsilon_{1}>0$ small enough, the map $z \rightarrow G(z)$ is a contraction on the Banach space of continuous maps $z:(0, \infty) \rightarrow X^{\alpha}$ equipped with the norm

$$
|z|_{\alpha, \beta}=\sup _{t \geqslant 0}\left\{|z(t)|_{X \alpha} e^{\beta t}\right\} \leqslant \rho,
$$

provided $a \in X_{2}(0),|a|_{X^{a}} \leqslant \rho / C_{24}$. Let $z^{*}(x, t ; a)$ be the unique fixed point of the above contraction. Then clearly $u^{*}(x, t ; a)=\bar{u}(x, t)+z^{*}(x, t ; a)$ is a solution of the differential system (5). To prove estimate (20) it will be shown that all solutions near $u^{*}$ are given in the above form. Following [1] the implicit function theorem will be a tool.

Given a solution of $(5),(6)$ if the initial function $b(x)$ is sufficiently close to $u_{0}(x)$ then the solution with $u(T, b)=b, t>T$ exists on the interval $[0,2 T]$. We shall show the existence of $\theta^{*}>0$ and $a^{*} \in X_{2}$, with $\left|\theta^{*}\right|$ and $\left|a^{*}\right|_{X^{\alpha}}$ small enough, such that

$$
u\left(x, \theta^{*} ; b\right)=u^{*}\left(x, 0 ; a^{*}\right), \quad t \geqslant \theta^{*}
$$

and therefore

$$
\left|u\left(x, t ; u_{0}\right)-\bar{u}\left(x, t-\theta^{*}\right)\right|_{X^{\alpha}} \leqslant \rho e^{-\gamma t}, \quad \gamma>0 .
$$

Let

$$
H(\theta, a ; b)=u(x, \theta ; b)-z^{*}(x, 0 ; a)+a-\bar{u}(x, t)-\theta \bar{u}_{t}^{\prime}(x, 0) .
$$

Then (69) is equivalent to

$$
a-\theta \bar{u}_{t}^{\prime}(x, 0)=H(\theta, a ; b)
$$


Let

$$
G(\theta, a ; b)=a-\theta \bar{u}_{t}(x, 0)-H(\theta, a ; b)
$$

Then

$$
\frac{\partial G}{\partial(a, \theta)}=\left(\begin{array}{cc}
\bar{u}_{t}^{\prime}(x, 0) & 0 \\
0 & I_{2}
\end{array}\right)
$$

is an isomorphism.

Applying the implicit function theorem yields the claimed statement. The theorem has been proved.

\section{REFERENCES}

[1] N.D. Alikakos, 'An application of the invariance principle to reaction-diffusion equations', J. Differential Equations 33 (1979), 201-225.

[2] L. Cesari, Functional analysis and periodic solutions to nonlinear differential equations, Vol. 1 (Interscience, New York, 1962).

[3] P. Constantin, C. Foias and R. Temam, 'On the large time Galerkin approximation of the Navier-Stokes equations', SIAM J. Numer. Anal. 21 (1984), 615-634.

[4] A. Dikansky, 'On the large time behavior of Galerkin approximations to the reaction-diffusion equations', Numer. Funct. Anal. Optim. (Submitted)

[5] A. Dikansky, 'Asymptotically stable stationary solutions of reaction-diffusion equations and their Galerkin approximations', Bull. Austral. Math. Soc. 47 (1993), 271-284.

[6] J. Hale, Ordinary differential equations (Wiley-Interscience, New York, 1969).

[7] D. Henry, Geometrical theory of semilinear parabolic equations, Lecture Notes in Math. 840 (Springer-Verlag, Berlin, Heidelberg, New York, 1981).

[8] P.E. Kloden, 'Asymptotically stable solutions of the Navier-Stokes equations and its Galerkin approximations', in Miniconference on Nonlinear Analysis (Canberra, 1984), Proc. Centre Math. Anal. Austral. Nat. Univ. 8 (Australian National University, Canberra), pp. 137-150.

[9] P.E. Kloedon, 'Asymptotically stable attracting sets for the Navier-Stokes equations', Bull. Austral. Math. Soc. 34 (1986), 37-52.

[10] A. Stokes, 'On the approximation of nonlinear oscillations', J. Differential Equations 12 (1972), 535-558.

[11] E.S. Titi, 'On a criterion for location stable stationary solutions to the Navier-Stokes equations', Nonlinear Anal. 11 (1987), 1085-1102.

[12] E.S. Titi, 'Critére numérique pour mettre en évidence des solutions periodiques des équations de Navier-Stokes', C.R. Acad. Sci. Paris Ser. I (1990).

Div of Mathematics and Computer Science

St John's University

300 Howard Ave

Staten Island NY 10301

United States of America 